University of Nebraska - Lincoln

DigitalCommons@University of Nebraska - Lincoln

Faculty Publications in Computer \& Electronics Electrical \& Computer Engineering, Department Engineering (to 2015)

2011

\title{
Timing accuracy of self-encoded spread spectrum navigation with communication
}

Won Mee Jang

University of Nebraska-Lincoln, wjang1@unl.edu

Follow this and additional works at: https://digitalcommons.unl.edu/computerelectronicfacpub

Jang, Won Mee, "Timing accuracy of self-encoded spread spectrum navigation with communication"

(2011). Faculty Publications in Computer \& Electronics Engineering (to 2015). 108.

https://digitalcommons.unl.edu/computerelectronicfacpub/108

This Article is brought to you for free and open access by the Electrical \& Computer Engineering, Department of at DigitalCommons@University of Nebraska - Lincoln. It has been accepted for inclusion in Faculty Publications in Computer \& Electronics Engineering (to 2015) by an authorized administrator of DigitalCommons@University of Nebraska - Lincoln. 


\title{
Timing accuracy of self-encoded spread spectrum navigation with communication
}

\author{
W. M. Jang \\ Department of Computer and Electronics Engineering, The Peter Kiewit Institute of Information Science, Technology \& \\ Engineering, University of Nebraska-Lincoln, Omaha, NE 68182, USA; email wjangl@unl.edu
}

\begin{abstract}
The author presents the timing accuracy of self-encoded spread spectrum (SESS) in navigation. SESS eliminates the need for traditional transmit and receive pseudo noise code generators. As the term implies, the spreading code is instead obtained from the random digital information source itself. SESS was shown to improve system performance significantly in fading channels. In this study, the authors investigate the timing accuracy of SESS in comparison to $m$-sequence and Gold code. SESS can be an excellent candidate for navigation with communication.
\end{abstract}

\section{Introduction}

Recent achievements in wireless technology could promote a new generation of radar networks, where the communication message is embedded into the radar waveform [1]. Global positioning system (GPS) also carries 50 bps navigation message through coarse acquisition (C/A) code and precise (P) code. In self-encoded spread spectrum (SESS) system the data bit information is used for generating the user spreading sequences [2]. At the transmitter, the current bit is spread by the output of a delay shift register that stores the previous $N$ bits, so that the current bit information would be used in the spreading operation of the next $N$ bits. The SESS spreading and despreading operation is shown in Figure 1. With iterative detection (ID), the estimate of the current bit can therefore be updated as the next $N$ bits are received. This results in an overall diversity gain. SESS ID or the chip-interleaved (CI) SESS ID was shown to improve the bit error rate (BER) performance significantly in fading channels as illustrated in Figure 2 [3]. Figure 3 shows simulation results of one- to five-user SESS CI with ID with multiple access interference cancellation $[4,5]$. The spreading gain of 64 for SESS and 63 for $m$-sequence is employed. We can observe that five-user SESS with interference cancellation demonstrates better performance than single-user $m$-sequence for the bit energyto- noise ratio $(E b / N o)$ greater than $2 \mathrm{~dB}$. The multipath channel impulse response of $\alpha_{1} \delta(\tau)+0.5 \alpha_{2} \delta(\tau-T / 2)$ is employed in Figure 4 where $\alpha_{i}$ are Rayleigh fadings, and $T$ is the bit duration. The second path is delayed by a half bit time with a half signal amplitude attenuation with respect to (w.r.t.) the first path. We can see that SESS performance is superior to $m$-sequence. In this paper, we investigate the possibility of SESS application in navigation. Unlike $m$-sequence or Gold code, SESS does not have a regulated auto- correlation. However, SESS provides the same timing accuracy with $m$-sequence or Gold code.

\section{Power spectral density (PSD) of SESS, $m$-sequence and Gold code}

Owing to the randomness of data bits, the PSD of SESS can be shown as

$$
S_{\mathrm{sess}}(\omega)=T_{\mathrm{c}} \operatorname{sinc}^{2}\left(\omega \frac{T_{\mathrm{c}}}{2}\right)
$$

where $T_{C}$ is the chip duration. Here we assume a rectangular pulse with the pulse width of $T_{c}$. For the timing accuracy estimation, the pulse bandwidth of $1 / T_{c}$ will be employed after passing through the low-pass filter for practical applications. The BER of the CI SESS ID was shown as [3]

$$
P_{\mathrm{b}}=\left[\frac{1}{2}(1-\mu)\right]_{k=0}^{N-1}\left(\begin{array}{c}
N-1+k \\
k
\end{array}\right)\left[\frac{1}{2}(1+\mu)\right]^{k}
$$

where the processing gain $N=T / T_{c}$. Here $\mu$ is defined as [17]

$$
\mu=\sqrt{\frac{\bar{\gamma}_{\mathrm{c}}}{1+\bar{\gamma}_{\mathrm{c}}}}
$$

where $\bar{\gamma}_{\mathrm{c}}$ is the average chip energy-to-noise ratio, $\bar{\gamma}_{\mathrm{c}}=2 E_{\mathrm{b}} /$ $\left(N N_{\mathrm{o}}\right) . E_{\mathrm{b}}$ is the bit energy and $N_{\mathrm{o}}$ is the one-sided noise PSD. Here we assume no self-interference that is negligible anyway at high signal-to-noise ratio (SNR) as shown in Figure 2. Self-interference effect because of incorrectly detected data bits at the receiver can also be removed with differential encoding [6]. Self-interference can be completely disregarded especially with 20 repetition codes as in GPS [7]. 


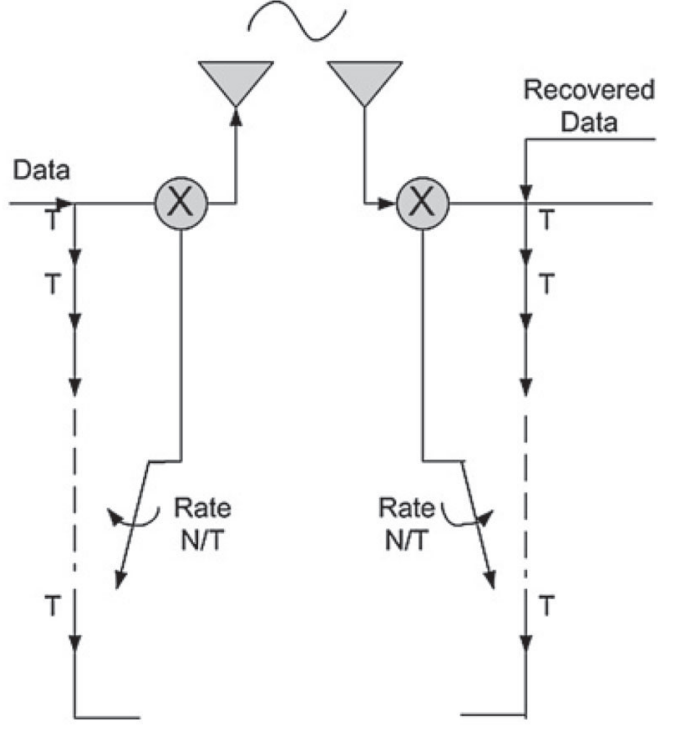

Figure 1. Self-encoded spread spectrum system structure.

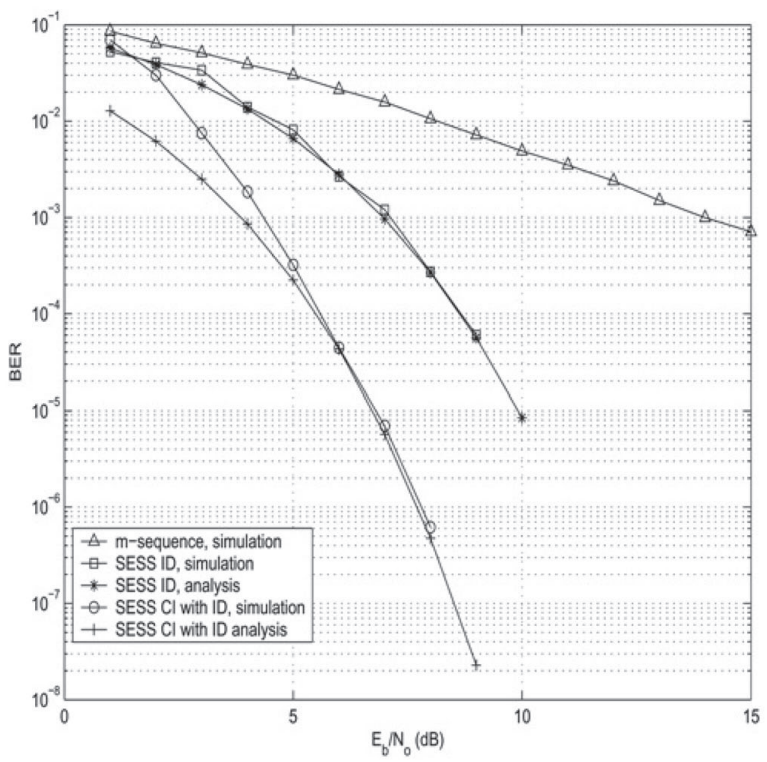

Figure 2. Analytical and simulation BER of SESS, single user, 64 chips/bit and Rayleigh fading.

A sequence of maximal period of $N=2^{n}-1$ generated by a linear feedback shift register of length $n$ is referred to as a maximal length sequence or $m$-sequence. The PSD of a $m$-sequence is shown as [8, pp. 600]

$$
\begin{aligned}
S_{\text {mseq }}(\omega)= & 2 \pi\left(1+\frac{1}{N}\right) \frac{1}{N} \sum_{n=-\infty}^{\infty} \delta\left(\omega-n \omega_{0}\right) \\
& \times \operatorname{sinc}^{2}\left(\frac{n \pi}{N}\right)-\frac{2 \pi}{N} \delta(\omega)
\end{aligned}
$$

where $\delta(\omega)$ is the unit impulse function, $\omega_{0}=2 \pi / T$ and $\operatorname{sinc}(x)=\sin (x) / x$.

Gold has shown that there exist preferred pairs of $m$-se-

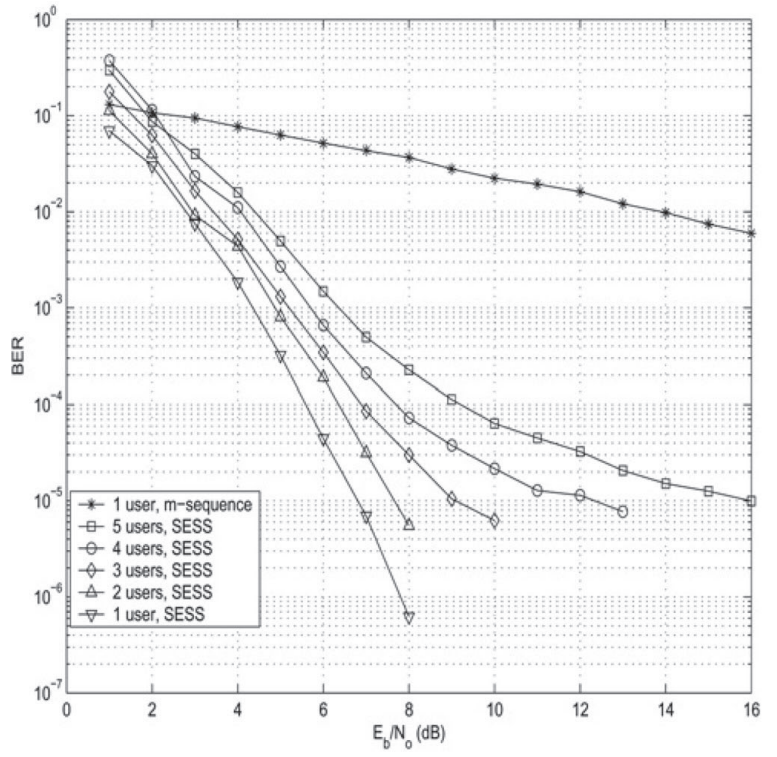

Figure 3. Simulation BER of SESS, chip interleaved ID, interference cancellation, one to five users, 64 chips/bit and Rayleigh fading.

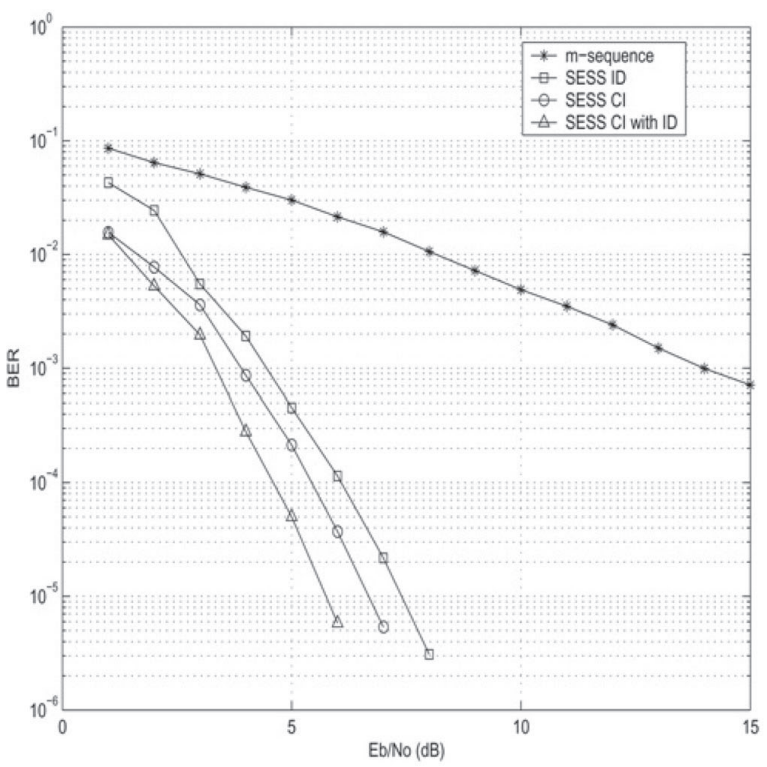

Figure 4. Simulation BER of SESS, single user, 64 chips-bit and frequency-selective multipath fading.

quences having three-level cross-correlation function when the degree $n$ is not an integer multiple of 4 [9]. The $m$-sequences with three-level cross-correlation function is referred to as the preferred pair. The product code generated by the preferred pair of $m$-sequences is referred to as a Gold code family. The PSD of a Gold code can be obtained as

$$
S_{m}(\omega)=\int_{-\infty}^{\infty} R_{m}(\tau) \exp \{-\mathrm{j} \omega \tau\} \mathrm{d} \tau
$$

where $R_{m}(\tau)$ is the autocorrelation function of the Gold code generated from the preferred pair with the second $m$ sequence shifted by $m$ chips. $S_{m}(\omega)$ is the corresponding PSD. The autocorrelation of a Gold code has a property of 
$R_{m}(i)=R_{m}(N-i)$ for $i=1, \ldots,(N-1) / 2$ [10]. Therefore the PSD component of the autocorrelation value $a_{k}^{m}\left(\left|a_{k}^{m}\right|, 1\right)$ with its delays $\pm d_{k i}^{m}\left(1 \leq d_{k i}^{m} \leq(N-1) / 2\right)$ can be expressed as

$$
\begin{aligned}
& s_{k i}^{m}(\omega)= a_{k}^{m} \frac{2 \pi}{T} \sum_{n=-\infty}^{\infty} \delta\left(\omega-n \omega_{0}\right) T_{\mathrm{c}} \operatorname{sinc}^{2}\left(n \omega_{0} \frac{T_{\mathrm{c}}}{2}\right) \\
& \times\left(\exp \left\{-\mathrm{j} d_{k i}^{m} T_{\mathrm{c}} n \omega_{0}\right\}+\exp \left\{\mathrm{j} d_{k i}^{m} T_{\mathrm{c}} n \omega_{0}\right\}\right) \\
&=2 a_{k}^{m} \frac{2 \pi}{N} \sum_{n=-\infty}^{\infty} \delta\left(\omega-n \omega_{0}\right) \operatorname{sinc}^{2}\left(\frac{n \pi}{N}\right) \cos \left(2 \pi d_{k i}^{m} \frac{n}{N}\right)
\end{aligned}
$$

Consequently, the PSD of a Gold code can be written as

$$
\begin{aligned}
S_{m}(\omega)= & \sum_{k=1}^{K_{m}} \sum_{i=1}^{k_{i}^{m}} s_{k i}^{m}+s_{0}=\frac{2 \pi}{N} \sum_{k=1}^{K_{m}} 2 a_{k}^{m} \\
& \times \sum_{i=1}^{k_{m}^{i}} \sum_{n=-\infty}^{\infty} \delta\left(\omega-n \omega_{0}\right) \operatorname{sinc}^{2}\left(\frac{n \pi}{N}\right) \cos \left(2 \pi d_{k i}^{m} \frac{n}{N}\right) \\
& +\frac{2 \pi}{N} \sum_{n=-\infty}^{\infty} \delta\left(\omega-n \omega_{0}\right) \operatorname{sinc}^{2}\left(\frac{n \pi}{N}\right)
\end{aligned}
$$

where $s_{0}$ is the PSD component because of the unit autocorrelation at $k T$ delay with an integer $k . K_{m}$ is the number of autocorrelation values with the second $m$-sequence shifted by $m$ chips. In fact, $K_{m}=3$ for all $m . k_{m}^{i}$ is the number of positive delays that attain the particular autocorrelation value of $\alpha_{k}^{m}$.

The average PSD of an ensemble of Gold codes can be found as

$$
\begin{aligned}
S_{\text {Gold }}(\omega) & =\frac{1}{N} \sum_{m=0}^{N-1} S_{m}(\omega) \\
& =\frac{1}{N} \sum_{m=0}^{N-1} \int_{-\infty}^{\infty} R_{m}(\tau) \exp \{-\mathrm{j} \omega \tau\} \mathrm{d} \tau \\
& =\int_{-\infty}^{\infty} \frac{1}{N} \sum_{m=0}^{N-1} R_{m}(\tau) \exp \{-\mathrm{j} \omega \tau\} \mathrm{d} \tau \\
& =\int_{-\infty}^{\infty} \bar{R}(\tau) \exp \{-\mathrm{j} \omega \tau\} \mathrm{d} \tau
\end{aligned}
$$

where the average autocorrelation $\bar{R}(\tau)$ is

$$
\begin{aligned}
\bar{R}(\tau)= & \frac{1}{N} \sum_{m=0}^{N-1} R_{m}(\tau)=\sum_{i=-\infty}^{\infty} \delta(\tau-i T) * \Delta\left(\frac{\tau}{2 T_{\mathrm{c}}}\right) \\
& +\frac{1}{N^{2}} \sum_{j=-\infty}^{\infty} \delta\left(\tau-j T_{\mathrm{c}}\right) * \Delta\left(\frac{\tau}{2 T_{\mathrm{c}}}\right) \\
& -\frac{1}{N^{2}} \sum_{k=-\infty}^{\infty} \delta(\tau-k T) * \Delta\left(\frac{\tau}{2 T_{\mathrm{c}}}\right) \\
= & \left(1-\frac{1}{N^{2}}\right) \sum_{k=-\infty}^{\infty} \delta(\tau-k T) * \Delta\left(\frac{\tau}{2 T_{c}}\right)+\frac{1}{N^{2}}
\end{aligned}
$$

where * indicates convolution. Here $\Delta(\tau / x)$ is a triangle pulse with the unit height and the width of $x$. We observe that $\bar{R}(\tau)$ is equal to the unity for $\tau=k T$ with an integer $k$, and is periodic with $T$. The result comes from the fact that the autocorrelation ensembles are cyclic shifted versions of the cross-correlation between $m$-sequences [10, equation (13)]. In addition, the summation of the cross-correlation for one period is equal to the unity [11, equation (3.5)]. Therefore we obtain the average PSD of Gold codes as

$$
\begin{aligned}
S(\omega)_{\mathrm{Gold}}= & \frac{2 \pi}{N}\left(1-\frac{1}{N^{2}}\right) \sum_{n=-\infty}^{\infty} \delta\left(\omega-n \omega_{0}\right) \\
& \times \operatorname{sinc}^{2}\left(\frac{n \pi}{N}\right)+\frac{2 \pi}{N^{2}} \delta(\omega)
\end{aligned}
$$

The explicit expression of the average PSD of Gold codes has not been found in literature to the best of the author's knowledge. In Appendix 1, we show that the timing accuracy of an individual Gold code with a different phase shift $m$ from (8) is asymptotically equal to the timing accuracy from the average PSD in (13).

\section{Timing accuracy of navigation}

The rms error (in second) in the measurement of the time delay of radar signal can be expressed as [12, p. 320; 13]

$$
\sigma_{\mathrm{t}}=\frac{1}{\beta \sqrt{\mathrm{SNR}}}=\frac{1}{\beta \sqrt{2 E_{\mathrm{b}} / N_{\mathrm{o}}}}
$$

where $\beta$ is called the effective bandwidth and is defined as

$$
\beta^{2}=\frac{\int_{-\infty}^{\infty} \omega^{2} S(\omega) \mathrm{d} \omega}{\int_{-\infty}^{\infty} S(\omega) \mathrm{d} \omega}=\frac{1}{S} \int_{-\infty}^{\infty} \omega^{2} S(\omega) \mathrm{d} \omega
$$

where $S(\omega)$ and $S$ are the signal PSD and the signal power, respectively. In Appendix 2, we show the asymptotic autocorrelation and PSD for SESS, Gold code and $m$-sequence as $N \rightarrow 1$. From (1) and (26), we can see that the asymptotic effective bandwidth is identical for SESS, Gold code and $m$-sequence applying the Riemann integral. Therefore the timing accuracy of the three sequences is the same asymptotically.

For multiuser and multipath channels, we can replace the SNR in (14) with signal-to-noise and interference ratio (SNIR). With $K$ interferers ( $K+1$ users) and $L$ multipaths, the SNIR for $m$-sequence or Gold code can be written as $[14,15]$

$$
\mathrm{SNIR}_{\mathrm{mG}}=\frac{R^{\mathrm{mf}}(0) 2 E_{\mathrm{b}} / N_{\mathrm{o}}}{1+\sum_{k=1}^{K} \sum_{l=1}^{L} \alpha_{k l}^{2}\left(1 / N^{2}\right)\left(2 E_{\mathrm{b}} / N_{\mathrm{o}}\right)}
$$

where $\alpha_{k l}$ is the fading of the $l$ th path of the $k$ th user. $R^{\mathrm{mf}}(0)$ is the power of the received signal of the desired user at the receiver input as shown in Appendix 3. The SNIR for SESS can be obtained as [16, p. 117]

$$
\mathrm{SNIR}_{\mathrm{sess}}=\frac{R^{\mathrm{mf}}(0) 2 E_{\mathrm{b}} / N_{\mathrm{o}}}{1+\sum_{k=1}^{K} \sum_{l=1}^{L} \alpha_{k l}^{2}(1 / N)\left(2 E_{\mathrm{b}} / N_{\mathrm{o}}\right)}
$$

because of its pure randomness in spreading sequences. Note that interference is larger in SESS since the autocorrela- 
tion or the cross-correlation is not regulated in SESS. The average timing accuracy in multiple access and multipath fading channels can be obtained as an expectation of (14) w.r.t. fadings and time delays using (16) and (17) and the PSD in multipath fading channels derived in (32) in Appendix 3.

\section{Numerical results}

The $m$-sequences corresponding to primitive polynomials, $(45)_{8}$ and $(67)_{8}$ in the octal notation, form a preferred pair with the code length $N=31$. The autocorrelation of the resultant Gold codes is three-valued with $-9,7$, and 21. Considering (8), for example with $m=1$, the autocorrelation values are $a_{1}=7 / 31, a_{2}^{1}=-1 / 31$, and $a_{3}^{1}=-9 / 31$. The delays of the autocorrelation value of $a_{1}^{1}$ are $d_{11}^{13}=5, d_{12}^{1}=7, d_{13}^{1}=10, d_{14}^{1}=$ 12 , and $d_{15}^{1}=14$. The PSD of a set of Gold codes for $m=1,3$, and 16 is plotted in Figure 5 using (8). Although the PSD plot is quite different for different $m$, all have the same rms value of the timing accuracy of $2.167 \mathrm{e}-7$, which is slightly smaller than $2.169 \mathrm{e}-7$ obtained from the average PSD in (13). The results are illustrated in Table 1 for SNR $=10 \mathrm{~dB}$. In calculation of the timing accuracy, we consider the bandwidth of $1 / T_{c}$ after the square pulse passing through the low-pass filter. Therefore we take the limit of the integration in (15) from $-2 \pi / T_{c}$ to $2 \pi / T_{c}$. We also show that the average of the individual PSD in (8) is equivalent to the PSD of the average autocorrelation in (13) already for $N=31$ as shown in Figure 6. Therefore we can use the PSD of the average autocorrelation to obtain the timing accuracy of Gold code. This result remarkably simplifies our discussion on the rms value of the timing accuracy of Gold code for a larger processing gain. In Table 2 we show the effective bandwidth $\beta$

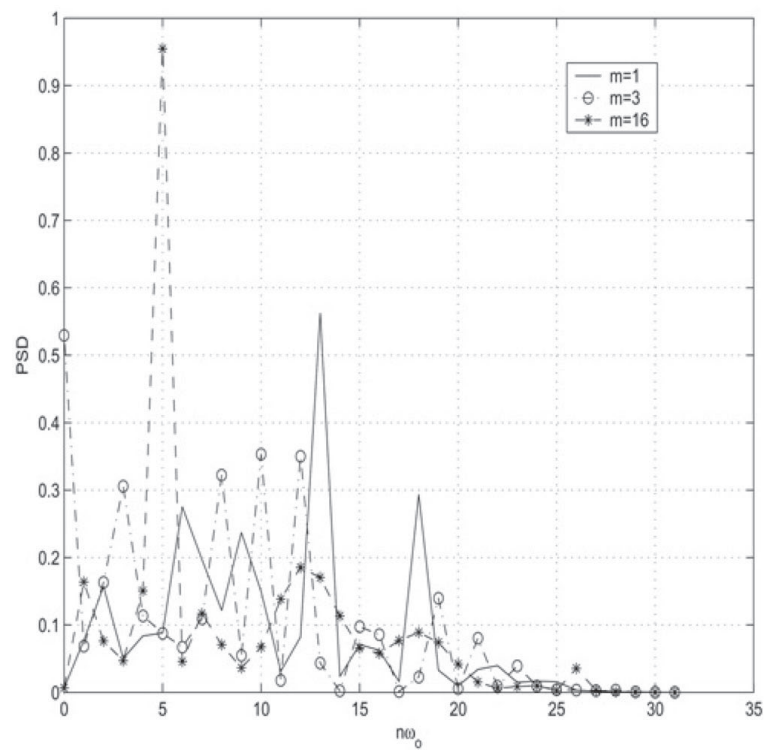

Figure 5. Power spectral densities of Gold codes, $\mathrm{m}=1$, 3, 16, $(45)_{8}$, and $(67)_{8}, N=31$.

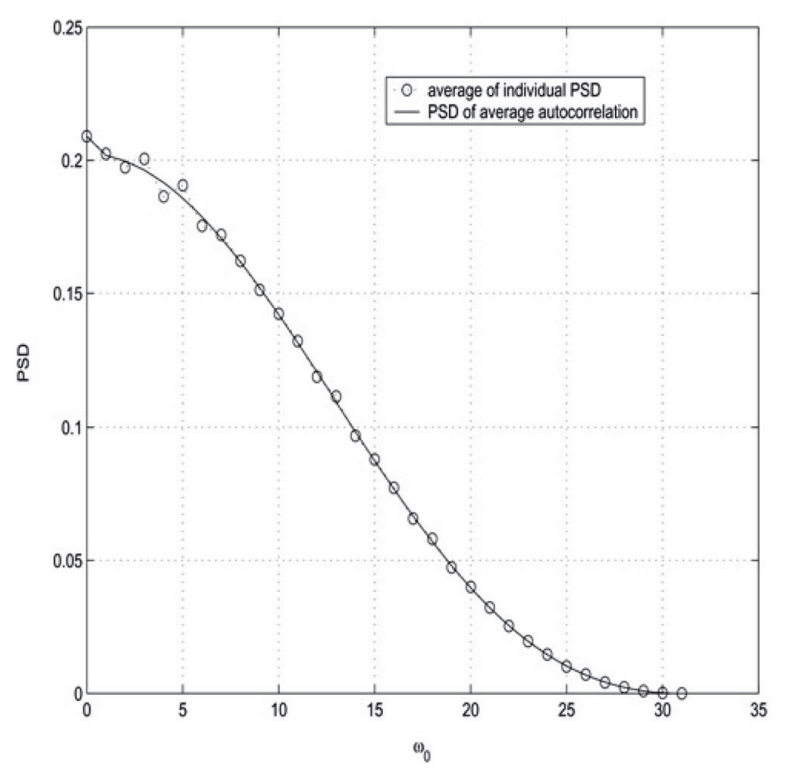

Figure 6. Average PSD of Gold codes using (8) and (13), $(45)_{8}$, and $(67)_{8}, N=31$.

and the rms value of the timing accuracy for $\mathrm{C} / \mathrm{A}$ code $(\mathrm{N}=$ $1023, T=1 \mathrm{~ms})$ and $\mathrm{P}$ code $(N=10,230, T=1 \mathrm{~ms})$ in GPS using SESS, $m$-sequences, and Gold codes. We assume additive white Gaussian noise (AWGN) channels for a GPS operating SNR of $10 \mathrm{~dB}$. A typical GPS receiver will update the display no more than twice a second, so the pulses from the correlator can be averaged over a period of a half second, which will decrease the rms error by $\sqrt{500}$ [7]. SESS, $m$-sequences, and Gold codes already show the same rms values of 6.6

Table 2. Rms error of timing accuracy $\left(\sigma_{\dagger}\right)$, AWGN channel, $\mathrm{SNR}=10 \mathrm{~dB}$, GPS

\begin{tabular}{lccc}
\hline$\beta, \sigma_{\mathrm{t}}$ & SESS & m-sequences & Gold codes \\
\hline$\beta(\mathrm{C} / \mathrm{A}$ code $)$ & $2.1533 \mathrm{e} 6$ & $2.1545 \mathrm{e} 6$ & $2.1533 \mathrm{e} 6$ \\
$\sigma_{\mathrm{t}}(\mathrm{C} / \mathrm{A}$ code $)$ & $6.5677 \mathrm{e}-9$ & $6.5641 \mathrm{e}-9$ & $6.5677 \mathrm{e}-9$ \\
$\mathrm{~b}(\mathrm{P}$ code $)$ & $2.1533 \mathrm{e} 7$ & $2.1534 \mathrm{e} 7$ & $2.1533 \mathrm{e} 7$ \\
$\mathrm{st}(\mathrm{P}$ code $)$ & $6.5677 \mathrm{e}-10$ & $6.5672 \mathrm{e}-10$ & $6.5677 \mathrm{e}-10$ \\
\hline
\end{tabular}

Table 3. Rms error of timing accuracy $\left(\sigma_{t}\right)$, multiple access and multipath channel, $\mathrm{K}=4$ (five users), $\mathrm{L}=2, \mathrm{t}_{1}=\mathrm{T} / 2, \alpha_{1}=1, \alpha_{2}$ $=0.5, \mathrm{SNR}=10 \mathrm{~dB}$ and GPS

\begin{tabular}{lccc}
\hline$\beta, \sigma_{\mathrm{t}}$ & SESS & m-sequences & Gold codes \\
$\beta(\mathrm{C} / \mathrm{A}$ code $)$ & $2.1348 \mathrm{e} 6$ & $2.1554 \mathrm{e} 6$ & $2.1533 \mathrm{e} 6$ \\
$\sigma_{\mathrm{t}}(\mathrm{C} /$ A code $)$ & $6.1035 \mathrm{e}-9$ & $5.8687 \mathrm{e}-9$ & $5.8745 \mathrm{e}-9$ \\
$\beta(\mathrm{P}$ code $)$ & $2.1388 \mathrm{e} 7$ & $2.1534 \mathrm{e} 7$ & $2.1529 \mathrm{e} 7$ \\
$\sigma_{\mathrm{t}}(\mathrm{P}$ code $)$ & $5.9322 \mathrm{e}-10$ & $5.8741 \mathrm{e}-10$ & $5.8753 \mathrm{e}-10$ \\
\hline
\end{tabular}

Table 1. Rms error of timing accuracy $\left(\sigma_{t}\right)$, Gold codes, $N=31, \mathrm{SNR}=10 \mathrm{~dB}$

\begin{tabular}{lcccccc}
\hline$\beta, \sigma_{\mathrm{t}}$ & $m=0$ & $m=1$ & $m=2$ & $m=3$ & $m=16$ & Average PSD \\
\hline$\beta(\mathrm{C} /$ A code $)$ & $6.52521 \mathrm{e} 4$ & $6.52521 \mathrm{e} 4$ & $6.52521 \mathrm{e} 4$ & $6.52521 \mathrm{e} 4$ & $6.52521 \mathrm{e} 4$ & $6.52135 \mathrm{e} 4$ \\
$\sigma_{\mathrm{t}}(\mathrm{C} /$ A code $)$ & $2.1673 \mathrm{e}-7$ & $2.1673 \mathrm{e}-7$ & $2.1673 \mathrm{e}-7$ & $2.1673 \mathrm{e}-7$ & $2.1673 \mathrm{e}-7$ & $2.1686 \mathrm{e}-7$ \\
\hline
\end{tabular}


and $0.66 \mathrm{~ns}$ for C/A code and P code, respectively. In Table 3 , we employ five-user multiple access system with the multipath channel impulse response of $\delta(\tau)+0.5 \delta(\tau-T / 2)$ since GPS signal from satellites in medium earth orbit (MEO) at a nominal altitude of $20,200 \mathrm{~km}$ is not significantly subject to fading. Table 3 presents 6 ns for C/A code, and 0.6 ns for P code for the three spreading codes. Comparing Tables 2 and 3 , we observe that multipath gain can outweigh multiple access and multipath interference because of the large processing gain.

\section{Conclusions}

SESS can be a good candidate for navigation with communication in fading channels such as indoor navigation, construction site or battle field navigation. SESS shows remarkable SNR enhancements in fading channels without compromising the timing accuracy compared with other traditional spreading codes such as $m$-sequence or Gold code. Moreover, if the large SNR gain in SESS can be available in the signal tracking stage, the time accuracy can be notably improved.

\section{Acknowledgments}

This work was supported by the contract award FA9550-081-0393 from the U.S. Air Force Office of Scientific Research. Thanks are due to Dr. J. A. Sjogren whose support has allowed the authors to investigate the timing accuracy of selfencoded spread spectrum for navigation.

\section{References}

1 Lellouch, G., Nikookar, H., On the capability of a radar network to support communications. IEEE 14th Symp. on Communication and Vehicle Technology, Benelux, 15 November 2007, pp. 1-5

2 Nguyen, L., Self-encoded spread spectrum and multiple access communications. IEEE Sixth Int. Symp. on Spread-Spectrum Tech. \& Appl., NJIT, New Jersey, 6-8 September 2000, pp. 394-398

3 Kim, Y.S., Jang, W.M., Kong, Y., Nguyen, L., Chip-interleaved selfencoded multiple access with iterative detection in fading channels, J. Commun. Netw., 2007, 9, (1), pp. 50-55

4 Patel, P., Holtzman, J., Performance comparison of a DS/CDMA system using a successive interference cancellation (IC) scheme and a parallel IC scheme under fading. Int. Conf. on Communications, IEEE ICC'94, May 1994, vol. 1, pp. 510-514

5 Divsalar, D., Simon, M.K., Improved CDMA performance using parallel interference cancellation. IEEE Military Communications Conf. MILCOM'94, October 1994, vol. 3, pp. 911-917

6 Kong, Y., Nguyen, L., Jang, W.M., Self-encoded spread spectrum modulation with differential encoding. IEEE Seventh Int. Symp. on Spread-Spectrum Tech. \& Appl., Prague, Czech, 2-5 September 2002, pp. $471-474$

7 Pratt, T., Bostian, C., Allnutt, J., Satellite communications (John Wiley \& Sons, 2003, 2nd edn.)

8 Ziemer, R.E., Peterson, R.L., Introduction to digital communication (Prentice Hall, 2001, 2nd edn.)

9 Gold, R., Maximal recursive sequences with 3-valued recursive crosscorrelation functions, IEEE Trans. Inf. Theory, 1968, IT-14, pp. 154-156

10 Rajan, J., On the histograms of the correlation functions of product codes. IEEE Int. Conf. on Acoustics, Speech, and Signal Processing, ICASSP'84, March 1984, vol. 9, Part 1, pp. 471-474

11 Sarwate, D.V., Pursley, M.B., Correlation properties of pseudoran- dom and related sequences, Proc. IEEE, 1980, 68, (5), pp. 593-619

12 Skolnik, M.I., Introduction to radar systems (McGraw Hill, 2001, 3rd edn.)

13 Mallinckrodt, A.J., Sollenberger, T.E., Optimum pulse-time determination, IRE Trans., 1954, 4, (PGIT-3), pp. 151-159

14 Gaudenzi, R.D., Elia, C., Viola, R., Band-limited quasi-synchronous CDMA: A novel satellite access technique for mobile and personal communication systems, IEEE J. Sel. Areas Commun., 1992, 10, (2), pp. 328-343

15 Jang, W.M., A Geometrical analysis of OFDM-CDMA with frequency offset, IEICE Trans. Commun., 2004, E87-B, (10), pp. 3021-3031

16 Verdu, S., Multiuser detection (Cambridge University Press, 1998)

17 Proakis, J.G., Digital communications (McGraw Hill, 2001, 4th edn.)

\section{Appendix 1}

\subsection{Independence of the timing accuracy of $S_{m}(\omega)$}

The PSD of a Gold code, Sm(v) in (8) can be rewritten as

$$
\begin{aligned}
S_{m}(\omega)= & \frac{2 \pi}{N} \sum_{n=-\infty}^{\infty} \delta\left(\omega-n \omega_{\mathrm{o}}\right) \operatorname{sinc}^{2}\left(\frac{n \pi}{N}\right) \\
& \times\left(1+\sum_{k=1}^{K_{m}} 2 a_{k}^{m} \sum_{i=1}^{k_{m}^{i}} \cos \left(2 \pi d_{k i}^{m} \frac{n}{N}\right)\right)
\end{aligned}
$$

Asymptotically as $N \rightarrow \infty$, the factor $2 \pi n / N$ in the cosine argument is uniformly distributed between $-\pi$ and $\pi$. Consequently, its asymptotic effective bandwidth can be obtained from (15) as

$$
\lim _{N \rightarrow \infty} \beta_{m}^{2}=\frac{\sum_{n=-\infty}^{\infty}(2 n \pi / T)^{2} \operatorname{sinc}^{2}(n \pi / N)}{\sum_{n=-\infty}^{\infty} \operatorname{sinc}^{2}(n \pi / N)}
$$

The result indicates that the timing accuracy of a Gold code is asymptotically independent of a chip shift of $m$. The effective bandwidth of the average PSD of Gold codes from (13) can be shown as

$$
\beta_{\mathrm{ave}}^{2}=\frac{\sum_{n=-\infty}^{\infty}(2 n \pi / T)^{2} \operatorname{sinc}^{2}(n \pi / N)}{\sum_{n=-\infty}^{\infty} \operatorname{sinc}^{2}(n \pi / N)+N /\left(N^{2}-1\right)}
$$

Therefore

$$
\lim _{N \rightarrow \infty} \beta_{m}^{2}=\lim _{N \rightarrow \infty} \beta_{\mathrm{ave}}^{2}
$$

\section{Appendix 2}

\subsection{Asymptotic equivalence of the timing accuracy of SESS}

The autocorrelation of SESS employing a rectangular pulse can be shown as

$$
R_{\mathrm{sess}}(\tau)=\Delta\left(\frac{\tau}{2 T_{\mathrm{c}}}\right)
$$

The autocorrelation of $m$-sequence and the average autocorrelation of Gold code are 


$$
\begin{aligned}
& R_{\mathrm{mseq}}(\tau)=\left(1+\frac{1}{N}\right) \Delta\left(\frac{\tau}{2 T_{\mathrm{c}}}\right)-\frac{1}{N}, \quad|\tau| \leq \frac{T}{2} \\
& R_{\mathrm{Gold}}(\tau)=\left(1-\frac{1}{N^{2}}\right) \Delta\left(\frac{\tau}{2 T_{\mathrm{c}}}\right)+\frac{1}{N^{2}}, \quad|\tau| \leq \frac{T}{2}
\end{aligned}
$$

respectively, with the period of $T$. Therefore we can see that asymptotically $(N \rightarrow \infty)$

$$
\begin{aligned}
\lim _{N \rightarrow \infty} R_{\text {mseq }}(\tau) & =\lim _{N \rightarrow \infty} R_{\mathrm{Gold}}(\tau) \\
& =\sum_{n=-\infty}^{\infty} \delta(\tau-n T) * \Delta\left(\frac{\tau}{2 T_{\mathrm{c}}}\right)
\end{aligned}
$$

The corresponding asymptotic PSD can be found as

$$
\begin{aligned}
\lim _{T_{\mathrm{c}} \rightarrow 0} S_{\mathrm{mseq}}(\omega) & =\lim _{T_{\mathrm{c}} \rightarrow 0} S_{\text {Gold }}(\omega) \\
& =\lim _{T_{\mathrm{c}} \rightarrow 0} \frac{2 \pi}{T} \sum_{n=-\infty}^{\infty} \delta\left(\omega-\frac{2 n \pi}{T}\right) T_{\mathrm{c}} \operatorname{sinc}^{2}\left(\omega \frac{T_{\mathrm{c}}}{2}\right)
\end{aligned}
$$

\section{Appendix 3}

\subsection{Autocorrelation and PSD in multipath fading channels}

With the multipath fading channel impulse response, $h(t)=$ $\Sigma_{i} \alpha_{i} \delta\left(t-t_{i}\right)$, the received signal without noise can be written as

$$
s(t)=\sum_{i} \alpha_{i} p\left(t-t_{i}\right)
$$

where $p(t)$ is the transmitted signal. $\alpha_{i}$ and $t_{i}$ are the $i$ th path fading and time delay, respectively. The corresponding auto- correlation can be obtained as

$$
\begin{aligned}
R^{\mathrm{mf}}(\tau)= & \int s(t) s(t-\tau) \mathrm{d} t \\
= & \int \sum_{i} \sum_{j} \alpha_{i} \alpha_{j} p\left(t-t_{i}\right) p\left(t-t_{j}-\tau\right) \mathrm{d} t \\
= & \sum_{i} \alpha_{i}^{2} R(\tau)+\sum_{i} \sum_{j \neq i} \alpha_{i} \alpha_{j} R\left(t_{j}-t_{i}+\tau\right) \\
= & \sum_{i} \alpha_{i}^{2} R(\tau)+\sum_{i} \sum_{j>i} \alpha_{i} \alpha_{j} R\left(t_{j i}+\tau\right) \\
& +\sum_{i} \sum_{j<i} \alpha_{i} \alpha_{j} R\left(-t_{j i}+\tau\right)
\end{aligned}
$$

where $t_{j i}=t_{j}-t_{i}$ and $R(\tau)$ is the autocorrelation of $p(t)$. The corresponding PSD can be written as

$$
\begin{aligned}
S^{\mathrm{mf}}(\omega)= & \sum_{i} \alpha_{i}^{2} S(\omega)+\sum_{i} \sum_{j>i} \alpha_{i} \alpha_{j} S(\omega) \exp \left\{\mathrm{j} t_{j i} \omega\right\} \\
& +\sum_{i} \sum_{j<i} \alpha_{i} \alpha_{j} S(\omega) \exp \left\{-\mathrm{j} t_{j i} \omega\right\} \\
= & \sum_{i} \alpha_{i}^{2} S(\omega)+2 \sum_{i} \sum_{j>i} \alpha_{i} \alpha_{j} S(\omega) \cos \left(t_{j i} \omega\right)
\end{aligned}
$$

where $S(\omega)$ is the PSD of $p(t)$. 